\title{
Mitteilungen des Verbandes Deutscher Gastroenterologen
}

Geschäftsstelle: Köln, Genter Straße 23, Telephon 512085

Worauf wartet eigentlich noch die Bundesärztekammer?

Von F. Matakas, Köln

Seitdem die Facharztbezeichnung für Magen- und Darmkrankheiten in der Bun-desrepublik auf der Liste der Fachärzte gestrichen wurde, sind 6 Jahre vergangen. Während dieser Zeit haben sich alle maßgebenden Stellen mit dieser Frage beschäf-tigt. Die Streichung dieses Fachgebietes hat eine Reaktion ausgelöst, die zu mannig-fachen Diskussionen auf wissenschaftlichem und berufspolitischem Gebiet führte. Alle auf diesem Gebiet maßgebenden Persönlichkeiten haben sich mit dieser Frage befaßt und ausnahmslos die Wiedereinführung dieser Facharztbezeichnung befür-wortet und gefordert. Unser Verband hat im Verlaufe zahlreicher Besprechungen mit den zuständigen Persönlichkeiten der Bundesärztekammer auf die nun ent-standene groteske Situation hingewiesen und um Rückgängigmachung der dama-ligen Fehlentscheidung gebeten. Die zuständigen Stellen der Bundesärztekammer haben unsere Argumente gewürdigt und uns zugesagt, den Wünschen unseres Verbandes Rechnung zu tragen.

Zwischenzeitlich hat sich unser Berufsverband mit den Berufsverbänden der Gastroenterologen der anderen 5 EWG-Länder zusammengefunden und gemeinsam mit ihnen die Sektion « Gastroenterologie » innerhalb der Europäischen Vereinigung der Fachärzte in Brüssel gegründet. Dabei wurde uns die Führung und der Vorsitz dieser Institution übertragen.

Diese Sektion hat im Rahmen des Weltkongresses für Gastroenterologie in Mün-chen im Mai 1962 ihre 1. Versammlung abgehalten und dabei eine Diskussion am runden Tisch durchgeführt, an der sich namhafte Vertreter aus den 6 EWG-Ländern beteiligten. Während dieser Diskussion, die sich vorwiegend mit der Ausbil-dung des künftigen Gastroenterologen befaßte, sprachen sich sämtlíche Teilnehmer für die Einführung bzw. Beibehaltung der Facharztbezeichnung für Gastroenterologie in alien Ländern der EWG aus. Dabei konnte man feststellen, daß die Bundesrepublik das einzige EWG-Land ist, in dem die Gastroenterologie als Fachgebiet nicht mehr anerkannt ist. Diese Tatsache wurde eben als grotesk bezeichnet, da alien Teilnehmern bekannt war, daß Deutschland das Geburtsland der Gastroenterologie ist und daß diese letztere erst von hieraus ihren Siegeszug über die ganze Welt begann.

Inzwischen wird in Brüssel auch innerhalb der Europäischen Vereinigung der Fachärzte weitergearbeitet. Bekanntlich wird im Jahre 1966/67 die sogenannte «Harmo-nisation» vollzogen. Die Fachärzte werden sich dann mit international gültigen Ausweiskarten in alien EWG-Ländern niederlassen können. Aus diesem Grunde wird jetzt schon die Angleichung in bezug auf Facharztbezeichnung und Ausbil-dungszeit durchgeführt. Im Mai dieses Jahres ist eine diesbezügliche Sitzung un-serer Sektion « Gastroenterologie » unter unserem Vorsitz in Paris vorgesehen. Wie soil es aber nun mit den deutschen Gastroenterologen werden? Obwohl die deut-schen Vertreter dieses Faches innerhalb der Europäischen Vereinigung der Fachärzte auf einstimmigen Beschluß den Vorsitz übernommen haben, sind sie nicht in der Lage, konkrete Angeben über die Ausbildungsform und -zeit der künftigen

212 Mitteilungen des Verbandes Deutscher Gastroenterologen 
Gastroenterologen zu machen, da das Fach der Gastroenterologie in der Bundes-republik immer noch nicht offiziell anerkannt ist. Die Zeit geht weiter. Selbst bei sofortiger Wiedereinführung dieses Fachgebietes werden die ersten Gastroenterologen in der Bundesrepublik erst in 4-5 Jahren mit ihrer Ausbildung fertig sein; also erst, nachdem die « Grenzen » zwischen den EWGLändern gefallen sind. Die Gastroenterologen in der Bundesrepublik werden täglich weniger, da kein Nachwuchs vor-handen ist. Logischerweise würde dann der Bedarf an solchen Spezialisten, der ohne Zweifel auch bei uns vorhanden ist (darüber habenwirja oft berichtet), aus den anderen Ländern der EWG gedeckt, zumal das Fach als solches vorläufig noch von zahlreichen praktisch tätigen KoUegen vertreten wird. Aus diesen Tatsachen geht eindeutig hervor, daß die Bundesärztekammer mit der Streichung dieses Fachgebietes einem großen Irrtum zum Opfer gefallen ist; denn unter den heute gegebe-nen Umständen wird man niemals erreichen können, daß die Gastroenterologie bei uns als Fach ausstirbt. Die getroffenen Maßnahmen werden höchstens dazu beitra-gen, daß die Vertreter dieses Faches in einigen Jahren aus den anderen Ländern der EWG zu uns kommen und sich hier niederlassen. Damit wird aber den deutschen Ärzten großes Unrecht getan, das auch mit den Bestimmungen unseres Grund-gesetzes nicht in Einklang zu bringen ist.

Unter diesen Umständen ist die passive Haltung der zuständigen Stellen der Bundesärztekammer nicht verständlich. Daher richten wir abschließend an diese Stellen die Frage:

«Worauf warten Sie eigentlich noch und was glauben Sie mit diesem Warten erreichen zu können?»

Die Zeit drängt, und an den Tatsachen können wir nicht mehr vorbeigehen. Es muß endlich gehandelt werden! Das Fach der Gastroenterologie muß auch bei uns wieder eingeführt werden. Wir bitten daher, unseren Antrag dem Deutschen Ärzte-tag noch in diesem Jahr zur Annahme vorzulegen. Die Sache ist längst spruchreif. Die Sektion « Gastroenterologie » der Europäischen Vereinigung der Fachärzte war-tet darauf, daß die Bundesärztekammer die entsprechenden Schritte unternimmt, um dann entsprechende Vorschläge zur vorgeschriebenen Angleichung machen zu können.

Assemblée générale statutaire de la section «Gastro-Entérologie»

de l'Union Européenne des Médecîns Spécialistes

en date du mardi 15 mai 1962 à Munich

La section de Gastro-entérologie de ГUnion Européenne des Médecins spécialistes (U.E.M.S.), constituée à Bruxelles en septembre 1960, a organise dans le cadre du Congrès de Munich, et à Toccasion de son Assemblée Générale statutaire biennale un colloque sur Tharmonisation de la formation du gastro-entérologue dans les six pays de la C.E.E.

Ce colloque a été organise avec la collaboration des professeurs Bartelheimer et Heinkel (Allemagne Fédérale), Ruyters et Van den Broucke (Belgique), Dubarry et Levrat (France), Haex (Hollande) et Gírolamí (Italie).

II a été preside par le DГ Matakas (Cologne), president de la section de Gastro-entérologie de ГU.E.M.S., assisté en qualité de conseiller et de modérateur par le DГ Massíon (Bruxelles) membre du Comité de Direction de ГU.E.M.S.

Le secretariat était assure par le DГ Crísmer (Liège) secretaire de la section de Gastroentérologie de TU.E.M.S.

Les professeurs invites à ce colloque eurent à répondre à un certain nombre de questions préparées par les DГs Matakas, Massíon et Crismer au nom de ГU.E.M.S.

En ouvrant le débat, le DГ Massíon rappela que le Traité de Rome prévoit le principe de la libre circulation et de la libre installation des personnes au sein de la C.E.E. 
Pour certaines professions, comme la profession médicale, il y a toutefois des restrictions prévues, à savoir une harmonisation préalable dans la formation du médecin généraliste ou spécialiste. En outre il est prévu que la pratique d'une spécialité dé-terminée ne pourra être libérée dans $\Gamma$ ensemble de la C.E.E. qu'à la condition que cette spécialité soit reconnue dans les 6 pays constituant la C.E.E. Or la Gastro-entérologie, qui a été considérée en Allemagne comme une spécialité autonome pendant 30 ans, a perdu sa qualification il y a 4 ans sous la pression des Internistes.

C'est la raison pour laquelle les organisateurs de ce colloque demandent à leurs interlocuteurs de répondre aux deux questions préalables suivantes:

$1^{\circ}$ Pensez-vous que la Gastro-entérologie doive être pratiquée comme une spécialité dans votre pays?

$2^{\circ}$ Estimez-vous qu'il convient qu'elle soit reconnue comme une spécialité dans tous les pays de la C.E.E ?

La réponse à ces deux questions a été unanimement positive, mais avec des nuances et parfois des restrictions.

Un enseignement officiel de la Gastro-entérologie est organise en France, en Hollande, en Italie et en Belgique fiamande, la durée de cet enseignement étant fort variable, allant de 2 ans en Italie à 5 ans en Hollande.

Les Allemands préféraient que les futurs gastro-entérologues acquièrent tout d'abord une large formation de médecine interne, d'une durée de 5 ans. Ensuite pendant deux ans il se consacrerait à $\Gamma$ étude particulière de la gastro-entérologie, ac-quérant ainsi le titre de médecin interniste avec le sous-titre de gastro-entérologue.

A ce système, certains voient le gros inconvénients d'un allongement démesuré des etudes.

Entrant ensuite dans le vif du sujet, les organisateurs du colloque posèrent suc-cessivement les 4 questions suivantes:

214 Mitteilungen des Verbandes Deutscher Gastroenterologen

$1^{\circ} \mathrm{La}$ formation du gastro-entérologue doit-elle commencer par des stages dans un service de mêdecine interne?

La réponse a été unanimement affirmative, mais avec des differences notables dans les modalités de ce stage, selon les pays.

Comme nous venons de le voir, les Allemands souhaitent 5 ans de mêdecine interne: les Hollandais exigent par contre un stage préalable de 3 ans en mêdecine interne. En France et en Italie, la situation est plus souple. En France la plupart des futurs spécialistes se recrutent parmi les internes des hôpitaux, qui, après la fin des etudes médicales normales, font trois à quatre ans supplémentaires de mêdecine interne. En Italie, les futurs spécialistes sont le plus souvent assistants pendant 4 ou 8 ans, dans un service de mêdecine, avant d'aborder les etudes de specialisation.

$2^{\circ} \mathrm{La}$ formation ultêríeure du gastro-entérologue doit-elle se faire dans un service specialise? Ici aussi la situation varie très fort selon les pays et même selon les universités.

En France, il y a une chaire de gastro-entérologie à Bordeaux, à Lille et à Marseille; il n'y en a pas à Paris ou à Lyon.

En Italie, il y a à Rome une Ecole Nationale de Gastro-entérologie. II en est de même, avec des modalités différentes, en Hollande, à Leyde, et en Belgique fla-mande à Louvain.

Ailleurs, la formation du gastro-entérologue se fait dans des services de mêdecine interne, dont Гactivité est orientée en ordre principal vers la gastro-entérologie, ou qui possède une section de gastro-entérologie. 
$3^{\circ}$ Queue doit être la durêe de la formation du gastro-entérologue?

Là aussi on trouve la plus grande diversité dans les réponses. En France, la durée officielle des etudes de specialisation est de trois ans, avec un examen severe de pa-thologie interne après la premiere année et un examen tout aussi severe de patholo-gie digestive à la fin des stages.

En Hollande les etudes de gastro-entérologie durent 5 ans, dont 3 ans de mêdecine interne et 2 ans de specialisation.

En Italie, les cours de $\Gamma$ Ecole Nationale de Gastro-entérologie durent 2 ans, mais exigent pour être suivis avec succès, une formation préalable de mêdecine interne très poussée.

A la section flamande à $\Gamma$ Université de Louvain, il existe un cours de gastro-entérologie d'une durée de 2 ans, venant après une formation interniste de deux à trois ans.

$4^{\circ}$ De queue manière concevez-vous la formation radíologíque du gastro-entérologue?

II y a une unanimité pour souhaiter et même exiger une formation radiologique très poussée du gastro-entérologue, certain désirant que cette formation commence dès le debut de stage en mêdecine interne.

II est cependant admis qu'il faut envisager avec souplesse et beaucoup de libéra-lisme le problème délicat de la formation radiologique technique.

II est concevable en effet que dans de très grandes villes et dans de très grands services, le gastro-entérologue s'associe à un radiologiste specialise et ne realise pas personnellement ses examens radiologiques.

Mitteilungen des Verbandes Deutscher Gastroenterologen 215

Par contre dans de plus petits services et dans de plus petites villes, il est par-faitement défendable pour le gastroentérologue de vouloir réaliser lui-même ses examens radiologiques. II devra dès lors acquérir les connaissances techniques né-cessaires. II n'est pas toutefois souhaitable de codifier d'une manière rigide la façon d'acquérir cette specialisation technique qui doit être laissée à Гappréciation du «Patron» de stage.

En resume, cette confrontation s'est révélée très utile et même très fructueuse. A la lumière des débats resumes ci-dessus on voit qu'il n'est pas encore possible d'unifier les méthodes de formation du Gastro-entérologue au sein de la C.E.E. Par contre, il se dégage de ce colloque des tendances convergentes qui avec le temps aboutiront sans nul doute à un réelle harmonisation. Compte rendu de $\Gamma$ Asse $\tau$ nblêe gênêrale statutaire du conseil de direction en date du mardi, 15 mai 1962, à Munich

Sont presents: DГ Matakas Dr Boom

DГ Crismer $\quad$ DГ Cornet

DГ Kumps Gírolami

DГ Schroeder

Est excuse: DГ Hertz

$1^{\circ}$ Allocution du President.

Le DГ Matakas souhaite la bienvenue aux délégués nationaux et rappelle que le Traité de Rome prévoit pour les médecins une harmonisation de Гexercice de la profession avant de permettre la libre circulation et la libre installation dans les 6 pays de la C.E.E. II rappelle aussi que la gastroentérologie est reconnue partout comme spécialité, sauf en Allemagne fédérale, ce qui est un obstacle à la liberation de cette spécialité dans le cadre de la C.E.E.

$2^{\circ}$ Rapport du Secretaire.

Le Dr Crismer rappelle tout d'abord que le comité de laison des gastro-entéro-logues de la C.E.E. fat créé à Bruxelles le 26 septembre 1960. Les statute provisoires ont été ratifies pour ГAUemagne fédérale par les DГ Matakas et Kaufmann, pour la Belgique par les DГ Crismer et 
Kumps, pour la France par les DГ Cachin et Bonnet, pour La Hollande par le DГ Boom et le Dr Hellemans, pour Cltalie par le Professeur Sotgiu, et le DГ Pisi et pour le Luxembourg par les Dr Hertz et Schwaal.

Conformément aux væux exprimés par le comité de direction de ГU.E.M.S. à Rome en avril 1961, puis à Amsterdam en octobre de la même année, le Comité de Liaison des GastroEntérologues de la C.E.E. a change d'appellation. II sera désor-mais désigné sous le nom de «Section de Gastro-Entérologie de ГU.E.M.S.».

Cette modification exige une modification du texte des statute, cette modification faisant $\Gamma$ objet du point 5 à Гordre du jour. Le DГ Crismer rappelle enfin les modifications survenues parmi les delegations des Unions Professionnelles Nationales: pour la France, le Prof. Cachin est remplacé par le Prof. Cornet et le DГ Bonnet par le DГ Guerre; pour la Hollande, le DГ Hellemans est remplacé par le DГ Schroeder et pour Гltalie, le Professeur Sotgiu et le DГ Pisi sont remplacés par le Professeur Girolamí et le DГ Cecchí.

216 Mitteilungen des Verbandes Deutscher Gastroenterologen

$3^{\circ}$ Rapport du Trésorier.

Le DГ Kumps rappelle que jusqu'à present les frais de secretariat, d'ailleurs mi-nimes, ont été couverts par $\Gamma$ Union Professionnelle Beige des Gastro-Entérologues.

Le DГ Kumps estime que le budget annuel de la section de Gastro-Entérologie de ГU.E.M.S. doit être de Tordre de 5000 francs beiges.

Le Professeur Cornet fait remarquer qu'il est difficile de demander à chaque so-ciété nationale, conformément à $\Gamma$ article 6 des statuts, une contribution proportion-nelle au nombre de ses membres. En France par exemple, il y a deux sortes de meni-bres: les gastro-entérologues «à part entière» et les gastro-entérologues «compé-tents», qualifies par le Conseil National de $\Gamma$ Ordre, mais n'exerçant pas exclusive-ment la gastro-entérologie. La cotisation de la France devrait-elle être calculée en fonction du nombre total de membres ou seulement en tenant compte des gastroentérologues n'exerçant que la spécialité ? Aussi, Tassemblée decide que, provisoire-ment, les sociétés nationales paieront une cotisation de 1000 francs beiges pour les trois grands pays (Allemagne Fédérale, France et Italie) de 500 francs beiges pour les deux pays moyens (Belgique et Hollande) tandis que Luxembourg ne paiera que 100 francs beiges.

$4^{\circ}$ Election des President, Secretaire et Trésorier pour $\Gamma$ Exercice 1962-1964.

Les Docteurs Matakas, Crísmer et Kumps sont réélus à Гunanimité, à main levée.

$5^{\circ}$ Modification des Statuts.

Sur proposition du Secretaire, les statuts de septembre 1960, sont modifies comme suit:

Art. 1, 2, 3, 5, 6, 7 et 12: Remplacer «Comité de Liaison des Gastro-Entérologues des Pays de la Communauté Economique européenne» par «Section monospécialisée de gastro-entérologie».

Art. 3: Remplacer «Les President et Secretaire des organisations profession-nelles nationales de gastro-entérologie» par «deux délégués des organisations professionnelles nationales de gastroentérologie».

Art. 5: Remplacer «dans la même ville que ГUnion Européenne des Médecins spécialistes» par «dans la ville où reside son secretaire». Art. 9: Ajouter «lorsque celui-ci a lieu en Europe». Art.

10: Ajouter «ou leur suppléant dûment mandaté».

Ces diverses modifications sont adoptés à $\Gamma$ unanimité des membres presents.

$6^{\circ}$ Programme d'activítés pour Гexercíce 1962-1964.

$1^{\circ}$ Determiner avec precision la situation de la gastro-entérologie dans les 6 pays de la C.E.E.

$2^{\circ}$ Organiser des enquêtes par questionnaires et contacts personnels sur les possi-bilités d'harmonisation de la pratique de la gastro-entérologie dans les 6 pays de la C.E.E. 
$3^{\circ}$ Faire des propositions dans ce sens à ГU.E.M.S. en la priant de les trans-mettre à la C.E.E. 